\title{
Factores asociados con abortos recurrentes en el Hospital Materno Infantil Showing Ferrari, Huánuco, 2018-2020
}

\author{
Factors associated with recurrent abortions at the Hospital Materno Infantil \\ Showing Ferrari, Huanuco, 2018-2020

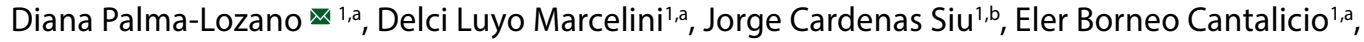 \\ Anibal Valentin Diaz Lazo ${ }^{1, c}$
}

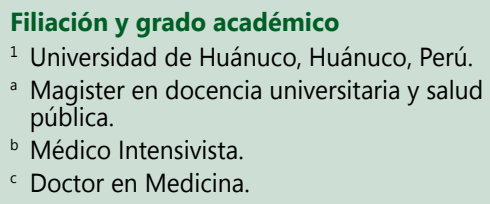

Fuentes de financiamiento

Autofinanciado.

Conflictos de interés

Los autores no presentan ningún conflicto de interés.

Recibido: 30-11-2020

Arbitrado por pares

Publicado en línea: 31-12-2020

Citar como

Palma-Lozano D, Luyo Marcelini D, Cardenas Siu J, Borneo Cantalicio E, Diaz Lazo AV. Factores asociados con abortos recurrentes en el Hospital Materno Infantil Showing Ferrari, Huánuco, 2018-2020. Rev Peru Cienc Salud. 2021; 3(1): 20-5. doi: https://doi. org/10.37711/rpcs.2021.3.1.240

Correspondencia

Diana Palma-Lozano

Telf.: 996264477

Email: diana.palma@udh.edu.pe

\section{RESUMEN}

Objetivo. Determinar los factores sociodemográficos y clínicos del aborto recurrente en el Hospital Materno Infantil Showing Ferrari, Huánuco, 2018-2020. Métodos. Se realizó un estudio observacional, retrospectivo y relacional en una cohorte. La población muestral fue 572 casos de mujeres con diagnóstico de aborto atendidas en el Hospital, entre octubre 2018 y diciembre 2020. Fueron excluidas las mujeres con embarazo ectópico y abortos completos al ingreso al Hospital. La técnica de recolección de datos fue por análisis documental a través del Sistema de Información Perinatal. Resultados. Se incluyen 572 (100\%) mujeres, un 45,3\% (259) de casos del grupo de 18 a 29 años, 56,5\% (323) de amas de casa y un 64,9\% (371) de conviviente. Respecto a las características clínicas, el 45,8 \% presentaron más de tres gestaciones, el $21 \%$ y $22,4 \%$ tuvieron anemia posprocedimiento e infección urinaria previa respectivamente y al 92,3\% le realizaron legrado. La frecuencia de aborto recurrente fue del $32 \%$. Los factores asociados con una mayor frecuencia para el aborto recurrente fueron: ser adolescente $p=0,000$, ama de casa $p=0,000$, soltera $p=0,000$, dos o más gestaciones $p=0,000$, trasfusión sanguínea $p=0,001$, anemia preprocedimiento $p=0,010$ y anemia posprocedimiento $p=0,034$. Conclusión. El aborto recurrente tuvo asociación significativa con ser adolescente, ama de casa, soltera, tener dos o más gestaciones, trasfusión sanguínea, anemia pre y posprocedimiento.

Palabras clave: aborto recurrente; aborto en adolescente; factores asociados (Fuente: DeCS-BIREME).

\section{ABSTRACT}

Objective. To determine the sociodemographic and clinical factors of recurrent abortion at the Hospital Materno Infantil Showing Ferrari, Huánuco, 2018-2020. Methods. An observational, retrospective and relational study was conducted in a cohort. The sample population was 572 women with a diagnosis of abortion attended at the Hospital, between October 2018 and December 2020. Women with ectopic pregnancy and complete abortions on admission to the hospital were excluded. The data collection technique was by documentary analysis through the Perinatal Information System. Results. A total of 572 (100\%) women were included, $45.3 \%$ (259) of cases were in the $18-29$ years age group, $56.5 \%$ (323) were housewives and $64.9 \%$ (371) were cohabiting women. Regarding clinical characteristics, $45.8 \%$ had more than three pregnancies, $21 \%$ and $22.4 \%$ had post-procedural anemia and previous urinary tract infection, respectively, and $92.3 \%$ had curettage. The frequency of recurrent abortion was $32 \%$. Factors associated with a higher frequency for recurrent abortion were: being an adolescent $p=0.000$, housewife $p=0.000$, single $p=0.000$, two or more gestations $p=0.000$, blood transfusion $p=0.001$, preprocedural anemia $p=0.010$ and postprocedural anemia $p=0.034$. Conclusion. Recurrent abortion has a significant association with being an adolescent, housewife, single, having two or more pregnancies, blood transfusion, pre- and post-procedural anemia.

Keywords: recurrent abortion; adolescent abortion; associated factors (Source: MeSH - NLM). 


\section{INTRODUCCIÓN}

El aborto es un grave problema de salud pública ${ }^{(1-4)}$ que presenta, en términos generales, vacíos de información como: la falta de una aproximación actualizada del número de abortos totales que ocurren en un país cada año o la inexistencia de análisis detallado sobre los costos económicos relacionados con la práctica clandestina (frecuentemente insegura) y los costos de las complicaciones asociadas a esto ${ }^{(5)}$. De otra parte, a pesar de las prohibiciones legales, muchas mujeres que viven en países con legislaciones restrictivas practican abortos, como resultado, estos se producen en clínicas clandestinas, sin condiciones higiénicas suficientes y sin el personal preparado para proveer el servicio de aborto seguro que proteja la vida de la mujer ${ }^{(6)}$. Las consecuencias que se generan son, por lo demás, médicas, culturales, religiosas, bioéticas, sociales, políticas y psicológicas.

En el periodo 2015-19, en el mundo hubo un promedio anual de 73,3 millones de abortos al año, lo que corresponde a una tasa mundial de 39 abortos por 1000 embarazos que se dieron entre las edades de 15 y 49 años ${ }^{(7)}$. Ramos et al. ${ }^{(8)}$ refieren que del total de abortos, el 50,9 \% fueron provocados en un promedio de 2,1 abortos por las propias mujeres. Ganatra et al. (9) describen que cada año, entre el 2010 y el 2014, 25,1 millones de abortos fueron inseguros; la proporción más alta fue en países en desarrollo frente a los países desarrollados (49,5\% frente a 12,5\%). Say et al. ${ }^{(10)}$, en un estudio que comprendió 60799 muertes maternas, indicaron que el $7,9 \%$ de los casos se debió a los abortos peligrosos.

En el Perú, ha podido determinarse indirectamente un número superior a 400000 abortos inducidos en el $2006^{(11)}$. Asimismo, no se cuentan con cifras sobre el impacto del aborto inseguro; sin embargo, se estima que cada año causa 28652 hospitalizaciones y 58 muertes ${ }^{(12)}$.

Adesse ${ }^{(13)}$ comenta que los estigmas del aborto varían de acuerdo a las distribuciones de las desigualdades de poder, a normas sexuales y reproductivas, a estereotipos de género, al imperativo de la maternidad, a la pureza sexual femenina, al instinto de cuidar a los demás y a considerar a las mujeres como seres inferiores. De Souza et al. ${ }^{(14)}$ refieren que las mujeres no solo deben sufrir un aborto, sino además ser presas de las indagaciones y cuestionamientos desde la perspectiva moral del aborto.

Los determinantes sociales de la salud desempeñan un papel importante en el resultado de los embarazos no deseados o no planeados; las mujeres en circunstancias sociales desfavorecidas tienen más probabilidades de experimentar un embarazo no deseado ${ }^{(15)}$. Para que una gestación termine en aborto, intervienen algunos factores de riesgo como: los antecedentes en el embarazo previo, edad materna avanzada, multiparidad, la forma en que finalizó el embarazo previo y la edad gestacional ${ }^{(16)}$. Mientras que en caso de un aborto recurrente prevalecen los factores epidemiológicos como la edad materna y los abortos previos ${ }^{(17)}$; los antecedentes personales 0 familiares, la genética de la madre y las infecciones ${ }^{(18)}$.

En los países donde el aborto ha estado restringido, la proporción de embarazos no deseados que terminaban en aborto aumentó y las tasas de embarazos no deseados eran más altas que en los países donde el aborto era generalmente legal ${ }^{(7)}$. En el Perú solo es legal cuando se realiza con el fin de salvar la vida de la mujer gestante ${ }^{(19)}$.

La pérdida recurrente del embarazo representa un reto para la medicina reproductiva ${ }^{(16)}$ y un problema de salud pública, por su extensión y las consecuencias que provienen de ella. En tal sentido, existe la necesidad de hacer estudios regionales que permitan caracterizar a fondo el fenómeno del aborto recurrente, seguro o inseguro, para dar respuestas ajustadas a las diferentes necesidades y para fortalecer los sistemas de información y fuentes primarias. Es por ello que se realizó este estudio con el objetivo de determinar los factores asociados con el aborto recurrente en el Hospital Materno Infantil Carlos Showing Ferrari (HMICSF) de Huánuco entre los años 2018 y 2020.

\section{MÉTODOS}

\section{Diseño del estudio}

Se realizó un estudio observacional, retrospectivo y relacional en una cohorte.

\section{Población muestral}

La población muestral fue de 572 mujeres con diagnóstico de aborto, quienes fueron atendidas en el HMICSF, en el Departamento de Huánuco, Perú. El periodo de estudio comprendió de octubre de 2018 a diciembre 2020. Se excluyó a las mujeres con diagnóstico de embarazo ectópico y con abortos completos al ingreso al Hospital.

\section{Recolección de datos}

La técnica de recolección de datos fue el análisis documental mientras que el instrumento empleado fue una ficha de registro que contenía las siguientes variables: edad, grado de instrucción, ocupación, estado civil, número de gestaciones, trasfusión sanguínea, anemia preprocedimiento, anemia posprocedimiento, infecciones urinarias previas y tipo de técnica para el tratamiento. Los datos fueron obtenidos del Sistema de Información 
Perinatal (SIP); sistema que permite el ingreso de todos los datos solicitados por la Organización Mundial de Salud (OMS) para producir información local, nacional y mundial.

\section{Definición de variables}

La variable aborto recurrente no tiene una definición clara y, por lo general se describe como la presencia de 2 o 3 abortos consecutivos ${ }^{(17,16)}$. La OMS ${ }^{(21)}$ refiere que el aborto puede causar daño posterior al acto que afecta a la calidad de vida o el bienestar, así como generar complicaciones tales como la muerte, hemorragias, infecciones o los traumatismos del aparato reproductor.

\section{Aspectos éticos}

Para la recolección de datos se obtuvo permiso de la responsable de investigación y capacitación del HMICSF, explicando los alcances de la investigación. El proyecto fue aprobado por el Comité de Ética de la Universidad de Huánuco. Los datos se manejaron con la confidencialidad y reserva del caso, según la declaración de Helsinki.

\section{Análisis de datos}

Los análisis estadísticos fueron realizados con el paquete estadístico STATA (Statacorp, TX, USA) versión 12.1. Se empleó la distribución de frecuencias y porcentajes para las variables cualitativas y el análisis bivariado chi cuadrado para los grupos independientes, considerando un $p<0,05$.

\section{RESULTADOS}

Se incluyeron 572 (100\%) mujeres con diagnóstico de aborto, de las cuales, resultaron en mayor porcentaje las del grupo de 18 a 29 años, con educación secundaria, ocupación ama de casa y de condición civil conviviente (ver tabla 1).

Respecto a las características clínicas, en el 45,8 \% de los casos, las mujeres presentaban más de tres gestaciones. En el 5,8 \% tuvieron trasfusión sanguínea, el $14,7 \%$ anemia preprocedimiento, el $21 \%$ anemia posprocedimiento y el $22,4 \%$ infección urinaria previa. Asimismo, a la mayoría de pacientes con aborto les fue realizado legrado (92,3\%) (ver tabla 2). La frecuencia de aborto recurrente en mujeres atendidas en el HMICSF fue del $32 \%$ (ver figura 1).

En el análisis bivariado (ver tabla 3) se halló asociación estadísticamente significativa entre el aborto recurrente y el ser adolescente $p=0,000$, ama de casa $p=0,000$,

Tabla 2. Características clínicas de mujeres con aborto atendidas en el HMICSF, Huánuco, 2018-2020 atendidas en el HMICSF, Huánuco, 2018-2020

\begin{tabular}{|c|c|c|}
\hline & fi & $\%$ \\
\hline \multicolumn{3}{|l|}{ Grupos de edad } \\
\hline 13 a 17 & 57 & 10,0 \\
\hline 18 a 29 & 259 & 45,3 \\
\hline 30 a más & 256 & 44,8 \\
\hline \multicolumn{3}{|l|}{ Grado de instrucción } \\
\hline Sin estudios & 27 & 4,7 \\
\hline Primaria & 116 & 20,3 \\
\hline Secundaria & 284 & 49,7 \\
\hline Superior universitaria & 145 & 25,3 \\
\hline \multicolumn{3}{|l|}{ Ocupación } \\
\hline Ama de casa & 323 & 56,5 \\
\hline Comerciante & 7 & 1,2 \\
\hline Agricultora & 2 & 0,3 \\
\hline Estudiante & 142 & 24,8 \\
\hline Otro & 98 & 17,1 \\
\hline \multicolumn{3}{|l|}{ Estado civil } \\
\hline Soltera & 102 & 17,8 \\
\hline Casada & 61 & 10,7 \\
\hline Divorciada & 35 & 6,1 \\
\hline Conviviente & 371 & 64,9 \\
\hline Viuda & 3 & 0,5 \\
\hline
\end{tabular}

\begin{tabular}{lcc}
\hline \multirow{2}{*}{ Características clínicas } & \multicolumn{2}{c}{$\mathbf{n = 5 7 2}$} \\
\cline { 2 - 3 } & $\mathbf{f i}$ & $\%$ \\
\hline Número de gestaciones & \\
Una & 134 & 23,4 \\
Dos & 130 & 22,7 \\
Tres & 46 & 8,0 \\
Más de tres & 262 & 45,8 \\
Trasfusión sanguínea & & \\
Sí & 33 & 5,8 \\
No & 539 & 94,2 \\
Anemia preprocedimiento & & \\
Sí & 84 & 14,7 \\
No & 488 & 85,3 \\
Anemia posprocedimiento & & \\
Sí & 120 & 21,0 \\
No & 452 & 79,0 \\
Infección urinaria previa & & \\
Sí & 128 & 22,4 \\
No & 444 & 77,6 \\
Tipo de técnica quirúrgica para el & & 7,7 \\
tratamiento & & 92,3 \\
AMEU & 44 & \\
Legrado & 528 & \\
\hline
\end{tabular}




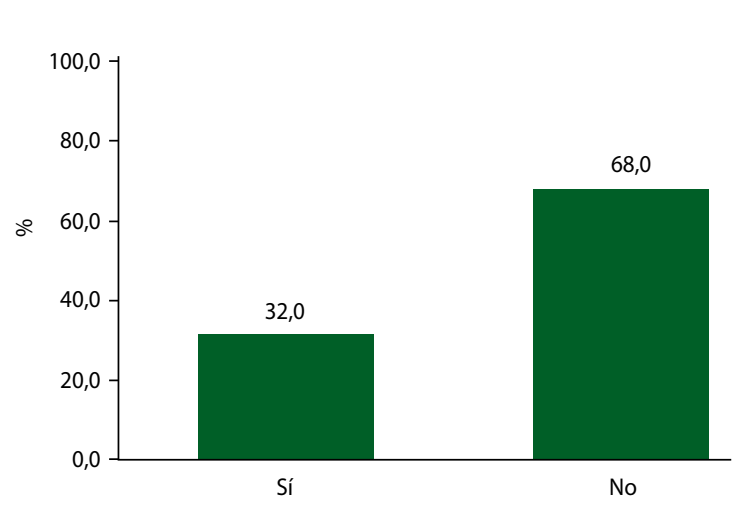

Figura 1. Abortos recurrentes en mujeres atendidas en el HMICSF, Huánuco, 2018-2020

soltera $p=0,000$, dos o más gestaciones $p=0,000$, trasfusión sanguínea $p=0,001$, anemia preprocedimiento $p=0,010$ y anemia posprocedimiento $p=0,034$.

\section{DISCUSIÓN}

El presente estudio parte de la necesidad de reconocer los factores del aborto recurrente, un problema de salud pública que, si bien podría ser tanto seguro como inseguro, por lo general ocasiona consecuencias físicas y psicológicas en la mujer, convirtiéndose en una dura experiencia para la paciente y todo un reto diagnóstico y terapéutico para el personal de salud tratante.

Es necesario mencionar, como parte de las características sociodemográficas, que la edad materna en la cual se presentaron un mayor número de casos de aborto fue de 18 años a más, similar a lo obtenido por Pérez et al. ${ }^{(20)}$, cuya edad promedio fue 24 más o menos 7 años. También se indica que, respecto al grado instrucción que tenían estas mujeres, el mayor porcentaje fue de secundaria y de ocupación ama de casa; aspectos que no fueron considerados por otros autores, empero es necesario mencionar pues este problema se suscita en personas con poca educación y en quienes se dedican al trabajo del hogar.

Los datos más resaltantes dentro de las características clínicas fueron que el 45,8 \% de las mujeres presentaban más de tres gestaciones, el 22,4 \% presentaba infección urinaria previa y el tipo de técnica quirúrgica para el tratamiento fue legrado en el 92,3\% de casos; similar a los resultados de Pérez et al. ${ }^{(20)}$ donde el $97 \%$ recibió el mismo procedimiento. Asimismo, es una cifra alarmante que el $32 \%$ del total presentaba aborto recurrente, datos que son semejantes al estudio retrospectivo de Cuba
Tabla 3. Factores asociados a abortos recurrentes en mujeres atendidas en el HMICSF, Huánuco, 2018-2020

\begin{tabular}{lcc}
\hline Factores & $\begin{array}{c}\text { Prueba chi } \\
\text { cuadrado }\end{array}$ & p valor \\
\hline Adolescente & 29,78 & 0,000 \\
Grado de instrucción bajo & 0,97 & 0,325 \\
Ama de casa & 21,14 & 0,000 \\
Soltera & 21,14 & 0,000 \\
Dos o más gestaciones & 82,32 & 0,000 \\
Trasfusión sanguínea & 10,53 & 0,001 \\
Anemia preprocedimiento & 6,58 & 0,010 \\
Anemia posprocedimiento & 4,47 & 0,034 \\
Infección urinaria previa & 0,72 & 0,395 \\
Legrado & 0,13 & 0,717 \\
\hline
\end{tabular}

y Ramírez ${ }^{(21)}$, donde el porcentaje de mujeres con dos abortos fue del $78 \%$, y del $20 \%$ con 3 .

Por consiguiente, ser adolescente en Huánuco (Perú) está asociado a aborto recurrente, sin embargo, Manzur (22) reporta resultados diferentes, indicando que las tasas de aborto clínico de 9-15\% se presentan en mujeres menores de 35 años y de 51-75\% en las mayores de 40 años. De igual forma lo manifiestan Rodríguez et al. ${ }^{(23)}$ o Cuba y Ramírez ${ }^{(21)}$. Esto conlleva a una reflexión pues, a pesar del conocimiento que tienen en la actualidad las adolescentes, siguen incurriendo en embarazos no deseados que puede terminar en aborto inducido y recurrente.

En relación al aborto recurrente y ser ama de casa, no se encontraron autores que abordan esta variable, sin embargo, el Ministerio de Salud y Protección Social de Colombia ${ }^{(6)}$ relata que la escolaridad, la actividad laboral, el número de hijos, la práctica anticonceptiva, el estrato social y la religiosidad, como factores determinantes del aborto, tiene aún un camino largo por recorrer, importante para contribuir en la construcción de las políticas públicas ajustadas a las condiciones reales de las mujeres que viven esta experiencia.

Del mismo modo, el resultado de la trasfusión sanguínea como factor asociado al aborto recurrente coincide con el estudio de Dónola ${ }^{(24)}$, en donde el 10,2 \% de mujeres requirieron dicho procedimiento. De otra parte, el grado de instrucción bajo y las infecciones urinarias previas no están asociados al aborto recurrente; este último aspecto queda reforzado por Mateo et al. ${ }^{(16)}$, quienes indican que, aunque no hay evidencia de que las infecciones provoquen pérdida recurrente del embarazo, se han identificado como causales de 
abortos espontáneos.

Asimismo, a la fecha de hoy no se cuenta con estadísticas locales acerca del aborto, más aún del aborto recurrente y los factores asociados. Por consiguiente, la fortaleza del estudio radica en que los resultados del mismo podrán contrastarse con los datos disponibles en la actualidad, con la finalidad de mejorar y replantear decisiones en la salud pública, tales como fomentar la prevención, fortalecer los programas sanitarios dedicados a mejorar la calidad de vida de las mujeres en edad fértil, reevaluar la dotación de anticonceptivos y trabajar la salud mental de las mismas.

Finalmente, se concluye que los factores asociados al aborto recurrente en el HMICSF de Huánuco, 20182020, fueron: ser adolescente, ama de casa, soltera, tener dos o más gestaciones, haber necesitado de trasfusión sanguínea o la anemia pre y posprocedimiento.

Se recomienda al HMICSF y a la Dirección Regional de Salud Huánuco (DIRESA), la ejecución de un proyecto viable y amigable para fortalecer acciones donde se genere conciencia en hombres y mujeres sobre el ejercicio autónomo de la sexualidad y la reproducción, así como sobre la maternidad y paternidad responsable y deseada. Del mismo modo, se recomienda fomentar redes de soporte de fácil acceso a través de las organizaciones sociales que trabajan por los derechos de las mujeres; especialmente en las áreas más distantes, donde las mujeres puedan encontrar información idónea.

\section{REFERENCIAS}

1. Leal M, Castelar M. Abortamento na Adolescência: Atuação de Psicólogas em Hospitais-Maternidade Públicos de Salvador, Bahia. Psicol Ciência e Profissão [Internet]. 2019; 39: 1-15 [Consultado 2021 Ene 23]. Disponible en: http://www.scielo.br/scielo.php?script=sci_arttext\&pid=S1414-98932019000100limita108\&tlng=pt

2. Santos A. A construção espírita do problema do aborto: ordem espiritual e discurso público. Reli Soc [Internet]. 2019; 39(3): 152-72 [Consultado 2021 Ene 23] Disponible en: http://www.scielo.br/scielo.php?script=sci_arttext\&pid=S0100-85872019000300152\&tlng=pt

3. Cruz V, Ferraz K, Souzas R, Gonçalvez B. Criminalização do aborto no Brasil e implicações à saúde pública. Rev Bioética. 2013; 21(3): 494-508.

4. De Souza R, Andreoni S. Fatores associados ao aborto induzido entre jovens pobres na cidade de São Paulo, 2007. Rev Bras Estud Popul [Internet]. 2012; 29(2): 409-19. [Consultado 2021 Ene 8] Disponible en: https:// www.scielo.br/pdf/rbepop/v29n2/a11v29n2.pdf

5. Monteverde M, Tarragona S. Abortos seguros e inseguros: Costos monetarios totales y costos para el sistema de salud de la Argentina en 2018. Salud Colect [Internet]. 2019; 9;15: e2275. [Consultado 2021 Feb 3] doi: $10.18294 /$ sc.2019.2275

6. Ministerio de Salud y Protección Social, Fondo de Pobla- ción de las Naciones Unidas. Determinantes del aborto inseguro y barreras de acceso para la atención de la interrupción voluntaria del embarazo en mujeres colombianas [Internet] [Consultado 2021 Feb 3] Disponible en: https://www.minsalud.gov.co/sites/rid/Lists/BibliotecaDigital/RIDE/DE/SM-Determ-aborto-inseguro.pdf

7. Bearak J, Popinchalk A, Ganatra B, Moller AB, Tunçalp Ö, Beavin $C$, et al. Unintended pregnancy and abortion by income, region, and the legal status of abortion: estimates from a comprehensive model for 1990-2019. Lancet Glob Heal [Internet]. 2020; 8(9): e1152-61 [Consultado 2021 Ene 10] Disponible en: https://www.thelancet. com/pdfs/journals/langlo/PIIS2214-109X(20)30315-6. pdf

8. Ramos I, Hawker S, Da Silva S, Schuch C. Aborto induzido em mulheres de baixa renda: dimensão de um problema. Cad Saude Publica [Internet]. 1991; 7(2): 251-66 [Consultado 2021 Ene 8] Disponible en: https://www.scielo.br/scielo.php?script=sci_arttext\&pid=S0102-311X1991000200009

9. Ganatra B, Gerdts C, Rossier C, Johnson BR, Tunçalp Ö, Assifi $A$, et al. Global, regional, and subregional classification of abortions by safety, 2010-14: estimates from a Bayesian hierarchical model. Lancet [Internet]. 2017; 390(10110): 2372-81 [Consultado 2021 Ene 11] Disponible en: https://www.thelancet.com/journals/lancet/ article/PIIS0140-6736(17)31794-4/fulltext

10. Say L, Chou D, Gemmill A, Tunçalp Ö, Moller A-B, Daniels $J$, et al. Global causes of maternal death: a WHO systematic analysis. Lancet Glob Heal [Internet]. 2014; 2(6): e323-33 [Consultado 2021 Ene 10] Disponible en: https://pubmed.ncbi.nlm.nih.gov/25103301/

11. Maradiegue E. Aborto como causa de muerte materna. Rev Peru Ginecol Y Obstet [Internet]. 2006; 52(3): 150-3 [Consultado 2021 Ene 21] Disponible en: http://www. redalyc.org/articulo.oa?id=79890103

12. Taype-Rondan A, Merino-Garcia N. Hospitalizaciones y muertes por aborto clandestino en Perú: ¿Qué dicen los números? Rev Peru Med Exp Salud Publica [Internet]. 2016; 33(4): 829 [Consultado 2021 Ene 21] Disponible en: https://rpmesp.ins.gob.pe/index.php/rpmesp/article/view/2573

13. Adesse L, Bonan C, Da Silva K, Matos V. Aborto e estigma: uma análise da produção científica sobre a temática. Cien Saude Colet [Internet]. 2016; 21(12): 3819-32 [Consultado 2021 Ene 8] Disponible en: http://www.scielo.br/scielo.php?script=sci_arttext\&pi$\mathrm{d}=\mathrm{S} 1413-81232016001203819 \&$ Ing $=p t \& t \operatorname{lng}=p t$

14. De Souza R, Fusco C. Comportamento do aborto induzido entre jovens em situação de pobreza de ambos os sexos - Favela México 70, São Paulo, Brasil, 2013. Reprodução Clim [Internet]. 2016; 31(1): 13-21 [Consultado 2021 Ene 9] Disponible en: http://dx.doi.org/10.1016/j. recli.2015.12.001

15. Fusco C, De Souza R, Andreoni S. Unsafe abortion: social determinants and health inequities in a vulnerable population in São Paulo, Brazil. Cad Saude Publica [Internet]. 2012; 28(4): 709-19 [Consultado 2021 Ene 10] Disponible en: http://www.scielo.br/scielo.php?script=sci_arttext\&pid=S0102-311X2012000400010\&ln$\mathrm{g}=\mathrm{en} \& \operatorname{tlng}=\mathrm{en}$

16. Mateo-Sánez HA, Mateo-Sánez E, Hernández-Arroyo L, Rivera-Ramírez P, Mateo-Madrigal M, Mateo-Ma- 
drigal V, et al. Recurrent pregnancy loss: A literature review [Pérdida recurrente del embarazo: Revisión bibliográfica]. Ginecol Obstet Mex [Internet]. 2016; 84(8): 523-34 [Consultado 2021 Ene 12] Disponible en: https://www.scopus.com/inward/record.uri?ei$d=2-s 2.0-85026635105 \&$ partner $I D=40 \& m d 5=33 b-$ ba0ecf62dcd33f850fa1eca8266af

17. Ávila S, Gutiérrez J. Aborto recurrente-revisión. Asoc Costarric Med Leg y Discip Afines - Edición Virtual [Internet]. 2017; 34 (1): 1-11 [Consultado 2021 Ene 12] Disponible en: https://www.scielo.sa.cr/pdf/mlcr/ v34n1/2215-5287-mlcr-34-01-226.pdf

18. Van Niekerk EC, Siebert I, Kruger TF. An evidence-based approach to recurrent pregnancy loss. S Afr J Obstet Gynaecol [Internet]. 2013; 19(3): 61 [Consultado 2021 Ene 13] Disponible en: http://hmpg.co.za/index.php/sajog/ article/view/1534

19. Gobierno Regional de Salud. Protocolo para el manejo de casos de interrupción legal del embarazo [Internet] [Consultado 2021 Ene 10] Disponible en: http://bvs.minsa.gob.pe/local/minsa/2228.pdf

20. Pérez-Arciniegas E, Godoy-Albornoz D, Quiroz-Figuera D, Quiroz-Figuera D, Tovar-Thomas C, Romero-Herrera A, et al. Aspectos clínico-epidemiológicos del aborto en un Hospital de Upata. Estado Bolívar-Venezuela. Rev Biomed [Internet]. 2016; 27(1): 3-9 [Consultado 2021 Feb 3] Disponible en: http://revistabiomedica.mx/index. $\mathrm{php} / \mathrm{revbiomed/article/view/33}$

21. Cuba H, Ramírez F. Edad Materna avanzada como factor de riesgo de aborto recurrente en el Hospital Belén de Trujillo entre el año 2013-2018. Acta Médica Orreguiana Hampi Runa. 2019; 19(1): 29-41 [Consultado 2021 Feb 3] Disponible file:///c:/users/investigador\%201/downloads/1452-5094-1-pb.pdf

22. Manzur A. Aborto recurrente. Rev Médica Clínica Las Condes [Internet]. 2010; 21(3): 416-23 [Consultado 2021 Ene 28] Disponible en: http://dx.doi.org/10.1016/S07168640(10)70553-8

23. Rodríguez N, Cala A, Nápoles J, Milán Y, Aguilar M. Factores de riesgo asociados al embarazo en adolescentes. Rev Inf Cientítfica [Internet]. 2018; 9(5): 945-54 [Consultado 2021 Feb 3]. Disponible en: http://scielo.sld.cu/scielo.php?script=sci_arttext\&pi$\mathrm{d}=$ S1028-99332018000500945\&lang=es

24. Dónola M. Características de la atención del a 2018 [Internet] Huacho: Universidad Nacional José Faustino Sánchez Carrión Facultad; 2020 [Consultado 2021 Ene 8]. Disponible: http://repositorio.unjfsc.edu.pe/handle/ UNJFSC/3961 